\title{
Design of Intelligent Power Supply Unit Based on Zigbee Technology
}

\author{
Hui Qi ${ }^{1, a^{*}}$, Ningyuan $\mathrm{Gao}^{2, \mathrm{~b}}$ and Yixiao Zhang ${ }^{3, \mathrm{c}}$ \\ ${ }^{1,2,3}$ No.100, Pingleyuan, Chaoyang District, Beijing, China \\ aquihui@bjut.edu.cn, bgcy5505@126.com, czyx9036@163.com
}

(College of Electronic Information and Control Engineering, Beijing University of Technology, Beijing 100124, China)

Keywords: Zigbee technology; LPC1768; Lwip; Gateway; RFID;

\begin{abstract}
Zigbee technology is a kind of bi-directional wireless communication technology characterized by low complexity, low power consumption, close distance, low speed and low cost. It is mainly used for data transmission with low power consumption, low transmission speed and short distance, as well as application of typical periodic data, low reaction time data and intermittent data transmission. This article presents a design of intelligent power supply unit system based on zigbee technology. The system is composed of end devices, zigbee wireless network, wireless / Internet gateway and server. The goal of the system is to manage power supply to equipments automatically, and to develop the safety level of the work environment. Finally this article gives an analysis of the management and maintenance of the system, so as to contribute to further studies.
\end{abstract}

\section{Introduction}

With the continuous development of information technology and automation technology, internet of things (IOT) as a new discipline is continually perfected. The term internet of things was first used by Kevin Ashton in 1999 [1]. Appearance of the IPV6 network protocol allows the unique network address number reached trillion level, enough to allocate 1000 IP addresses per square meter on the earth, but also makes the dream of "all objects be linked" come true [2]. Internet of things is widely used throughout many areas including intelligent transportation, public safety, home security, intelligent fire alarm, smart home, military reconnaissance, etc [3]. In the foreseeable future, the internet of things will be applied to everyone's life. This article presents an intelligent power supply unit network based on zigbee networks and RFID technology, which is designed based on the idea of internet of things, to achieve the goals of remote control and automatic recording ability for the power supply system, and by setting the maximum current / voltage limit to protect the safety of the equipments. The intelligent power supply unit network can be widely used in factories, companies, laboratories and other work environments.

\section{Introduction to Overall Design}

Intelligent power supply unit network includes end devices, zigbee / Lwip gateway, server and upper computer. Every component connected to each other via zigbee wireless sensor networks and Internet. The basic functions of the end device is to collect the current, voltage, power and other parameters of the equipments and upload the data to server and upper computer immediately. In addition to storing data collected by the end devices, the upper computer could also send management commands and data collecting commands to end devices to read user ID, IP address and other parameters of the equipments. In addition, the upper computer can also send commands to set the maximum current / voltage values of the end devices. When the supply current / voltage exceeds the maximum limits, the end device will automatically cut off the power and send an exception report to upper computer, in order to protect the safety of the equipment. 


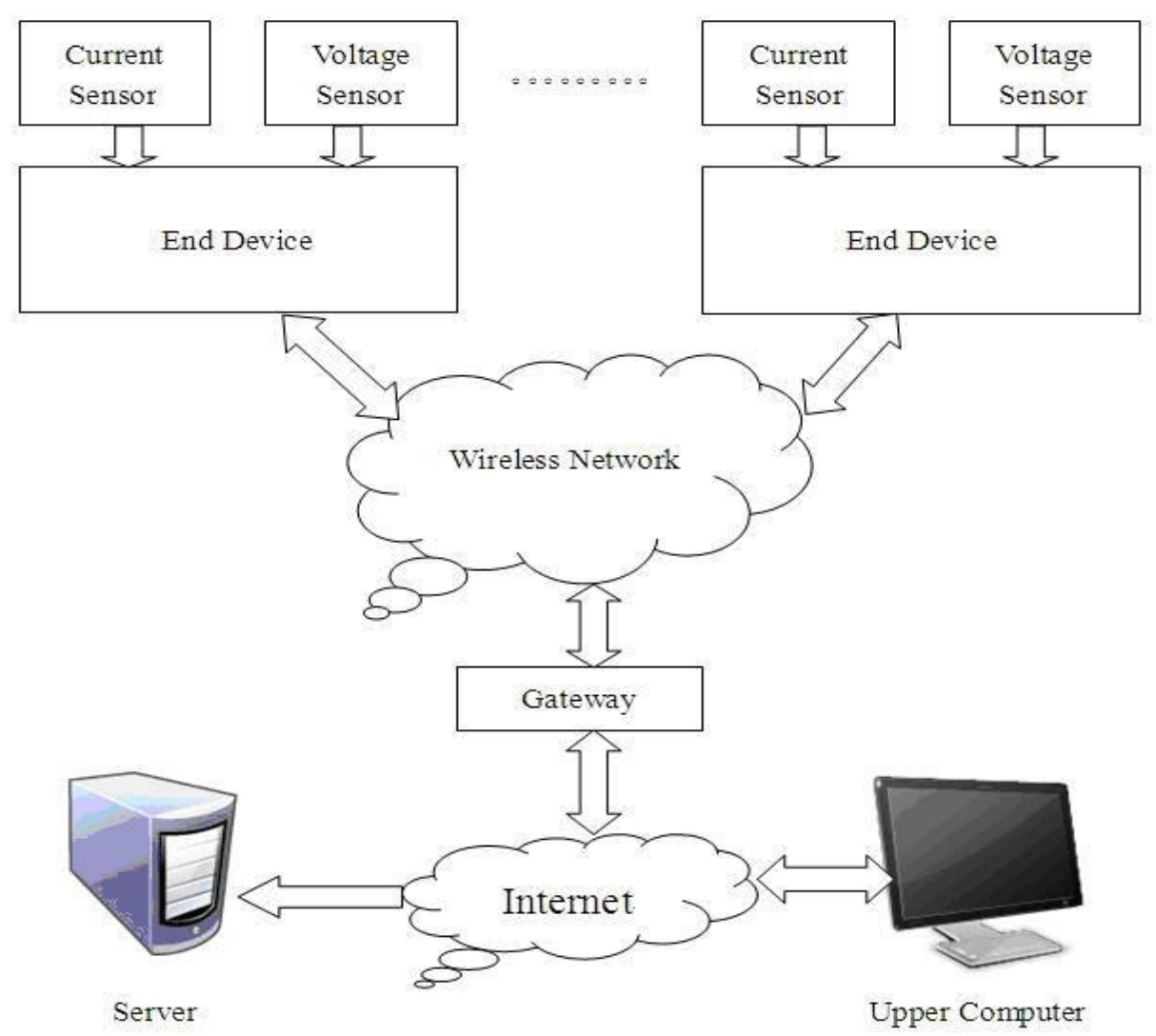

Figure. 1. The overall block diagram of the intelligent power supply unit network

\section{Design of the End Device of Intelligent Power Supply Unit Network}

Design and Implementation of Hardware. The end device includes a main controller, a current / voltage data collecting nodes, a RFID reader and a zigbee end device.

The type of the main controller is LPC1768 of NXP company. LPC1768 is designed based on Cortex-M3 core, with 512KB of Flash memory, $64 \mathrm{~KB}$ of data memory, Ethernet MAC, 8-channel DMA controller, 2 CAN channels, SPI interfaces, motor control PWM, 4 general purpose timers, ultra-low power RTC with an independent battery and 70 general-purpose I / O pins [4].

The type of the energy metering chip is ATT7022. ATT7022 is a high-precision three-phase power metering chip used for three-phase three-wire and three-phase four-wire applications. ATT7022 integrates sigma-delta ADC, voltage reference circuit and frequency measurement digital signal processing circuits. It is capable of measuring current, voltage, power, phase angle, frequency and other parameters [5].

RFID (radio frequency identification) is a technology using radio frequency signals through space to achieve non-contact information transmission, and to achieve the purpose of automatic identification technology by the information conveyed [6]. A typical RFID system consists of four parts: tags, readers, middleware and control computers, wherein the electronic tag is the carrier of RFID data, the reader is mainly used to read the electronic tag information and upload it to the control computer [7].

The type of the zigbee end device is CC2530 of Texas Instruments. CC2530 is a SOC solution designed based on IEEE802.15.4 and RF4CE. It can be used to establish a strong wireless network at a very low cost of materials [8]. CC2530 integrates a RF transceiver, an industry-standard enhanced $8051 \mathrm{CPU}$, a programmable Flash, 8KB RAM and other powerful features [9]. 


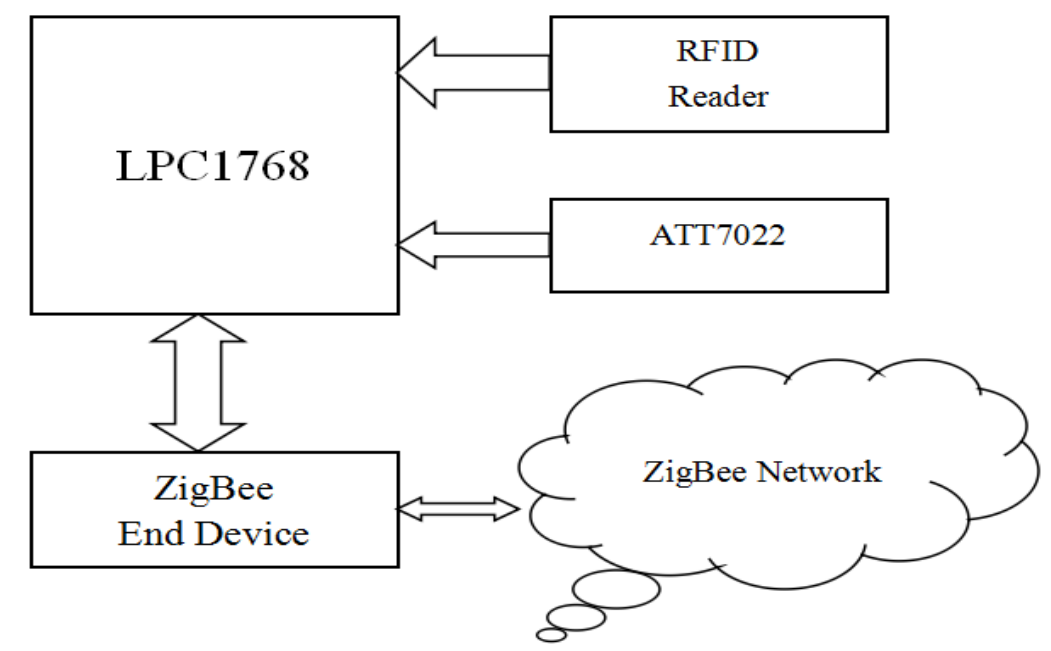

Figure. 2 Block diagram of end device

Design and Implementation of Software. The software of the end device is designed based on c language and Lwip network protocol, the main functions of the program includes:

a. Real-time data collected and uploaded to the server.

b. Accept commands from the upper computer and set the parameters of the end device including IP address, user ID, the maximum current / voltage.

c. Record the time of On / Off of the equipment and user ID, upload the record to the server after the power is off.

d. Accept commands from the upper computer to turn on / off the equipment.

e. When the measured value exceeds the maximum current / voltage limit, the program automatically cut off the power supply to the equipment, then upload the exception record to the server.

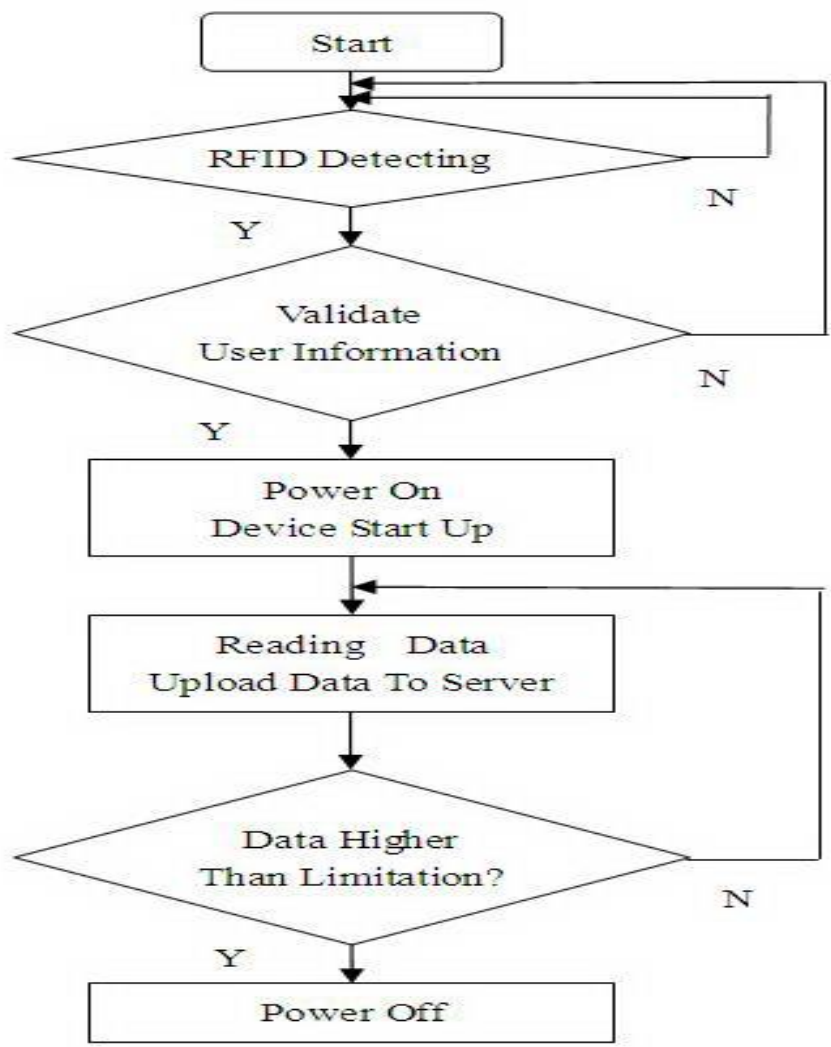

Figure. 3 The flow diagram of the software 


\section{Design of the zigbee / Lwip Gateway}

The gateway contains a main controller, a zigbee coordinator and an ethernet transceiver. The controller is still LPC1768, zigbee coordinator is CC2530 coordinator module. The type of Ethernet transceiver is SMSC company's LAN8710 chip, which is a high-performance low-power 10 BASE-T / 100 BASE-TX Ethernet transceiver specifically designed for consumer electronics, industrial and enterprise applications [10]. LAN8710 has the smallest footprint, makes the power consumption $40 \%$ lower than other transceiver.

The block diagram of the gateway is shown in Figure 4, the end devices upload the measured data to the coordinator through zigbee wireless network, the data is then transferred to the LPC1768 chip via the serial port, the gateway program converts the data and upload it to the server by Internet. The commands from the upper computer is transmitted via the opposite direction.

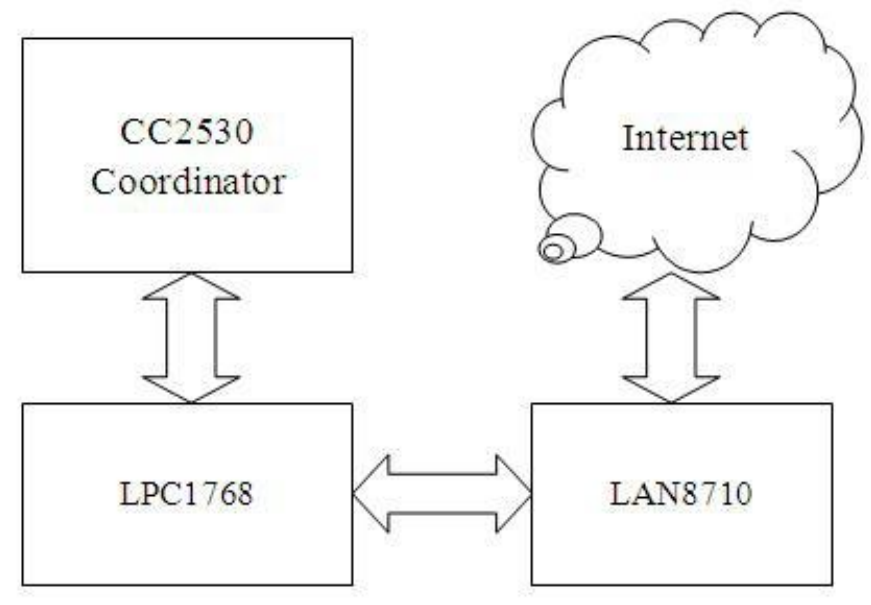

Figure. 4 Block diagram of gateway

\section{System Test Results and Analysis}

The system is tested under simulated conditions of work environment. Experiments show that the system is running in a stable condition, the end device could continually upload data and receive commands from the upper computer. There is no failure during the test.

The test of zigbee wireless network for link quality includes two parameters: network latency and packet loss rate. Run TI SmartRF Studio 7 software on Wuxianlong IOT1000 wireless network protocol analyzer to test the CC2530 wireless network. Results of the test are calculated as follows: the average network latency is about $15 \mathrm{~ms}$; packet loss rate is less than $1 \%$. The parameters fully meet the application requirements.

\section{Conclusion}

Intelligent power supply unit network consists of end devices, advanced zigbee wireless network and gateway achieves an automated and intelligent equipment power supply system. The entire system combines the advantages of low-cost and stability, has a broad prospects of application.

\section{References}

[1] Kevin Ashton, That 'Internet of Things' Thing, In:RFID Journal, 22 July 2009.

[2] P.Wei, Internet of things set off a new wave of information technology revolution, LOGISTICS\& MATERIAL HANDLING, Vol 14, No.11, 42—45, 2009. 
[3] Ying J, The Research and Design of Non-contact IC Card [D], Shanghai: Shanghai Institute of Metallurgy (SIM), 2000: 8-19.

[4] Ji W, Yang D, Hong G and Ge L: The research of RFID wireless network based on ZigBee technology. International Conference on Computational and Information Sciences (2013, June). p. 1392.

[5] Alharbe N, Atkins A S and Akbari A S: Application of ZigBee and RFID Technologies in Healthcare in Conjunction with the Internet of Things[C]. Proceedings of International Conference on Advances in Mobile Computing \& Multimedia (ACM, 2013). p.191.

[6] Patton M, Gross E and Chinn R: Uninvited Connections: A Study of Vulnerable Devices on the Internet of Things (IoT). Intelligence and Security Informatics Conference (JISIC, 2014). p.232.

[7] Yang F and Yan C: Design of WSN gateway based on ZigBee and TD. Electronics and Information Engineering (ICEIE, 2010). P.76.

[8] Wang Y and Zhang X: Design of a ZigBee-Ethernet Gateway. Electronic Science and Technology, 2012, 10: 037.

[9] Zhang C, Zhang M and Su Y: Smart home design based on ZigBee wireless sensor network. Proceedings of the 7th International ICST Conference on Communications and Networking(China, 2012). p.463.

[10]Chen X P and Jiang X: Design of the Wireless Power Acquisition Node Based on the LONWORKS and ZIGBEE Technology. Advanced Materials Research. 2012, 433: 7452-7457. 